\title{
Sequential Index Structure for Content-Based Retrieval
}

\author{
Maciej Zakrzewicz \\ Poznan University of Technology \\ Institute of Computing Science \\ Piotrowo 3a, 60-965 Poznan , Poland \\ mzakrz@Cs.put.poznan.pl
}

\begin{abstract}
Data mining applied to databases of data sequences generates a number of sequential patterns, which often require additional processing. The post-processing usually consists in searching the source databases for data sequences which contain a given sequential pattern or a part of it. This type of content-based querying is not well supported by RDBMSs, since the traditional optimization techniques are focused on exact-match querying. In this paper, we introduce a new bitmap-oriented index structure, which efficiently optimizes content-based queries on dense databases of data sequences. Our experiments show a significant improvement over traditional database accessing methods.
\end{abstract}

Keywords: sequential pattern discovery, index structures, databases 


\section{Introduction}

Mining of sequential patterns, a fundamental data mining method, consists in identifying trends in databases of data sequences (containing collections of records over a period of time)[AS95]. A sequential pattern represents a frequently occurring subsequence, i.e. a data sequence which is often contained in data sequences in the database. An example of a sequential pattern that holds in a video rental database is that customers typically rent "Star Wars", then "Empire Strikes Back", and then "Return of the Jedi". Note that 1. these rentals need not be consecutive , and 2. during a single visit, a customer may rent a set of videos, instead of a single one. Discovered sequential patterns are usually used as input for upper-level data mining methods: prediction, characterization, classification, clustering, etc.

Post-processing of discovered sequential patterns usually consists in searching the source databases for data sequences which contain a given sequential pattern (or a part of it). For example, when we discover an interesting sequential pattern in the video rental database, we would probably like to find all customers, who satisfy (contain) the pattern. A similar searching method can be used when we store discovered sequential patterns in databases and then retrieve them by contents, e.g. searching all sequential patterns which contain the subsequence "Star Wars" $\rightarrow$ "Return of the Jedi". We will refer to these types of searching as to contentbased sequence retrieval.

In most cases, data sequences (and discovered sequential patterns) are stored in relational, $S Q L$-accessed databases. Let us consider the following example of using the relational approach to content-based sequence retrieval. Assume that the relation $R(S I D, T S, L)$ stores data sequences. Each tuple contains the sequence identifier (SID), the timestamp $(T S)$, and the item $(L)$. Our example relation $R$ describes three data sequences: $\{\mathrm{A}, \mathrm{B}\} \rightarrow\{\mathrm{C}\} \rightarrow\{\mathrm{D}\}, \quad\{\mathrm{A}\} \rightarrow\{\mathrm{E}, \mathrm{C}\} \rightarrow\{\mathrm{F}\}$, and $\{\mathrm{B}, \mathrm{C}, \mathrm{D}\} \rightarrow\{\mathrm{A}\}$. Let the searched data subsequence be: $\{\mathrm{A}\} \rightarrow\{\mathrm{E}\} \rightarrow\{\mathrm{F}\}$. Figure 1 gives the relation $R$ and the $S Q L$ query, which implements the content-based sequence retrieval problem.

\begin{tabular}{lll} 
SID & TS & L \\
\hline 1 & 1 & A \\
1 & 1 & B \\
1 & 2 & C \\
1 & 3 & D \\
2 & 1 & A \\
2 & 2 & E \\
2 & 2 & C \\
2 & 3 & F \\
3 & 1 & B \\
3 & 1 & C \\
3 & 1 & D \\
3 & 2 & A
\end{tabular}

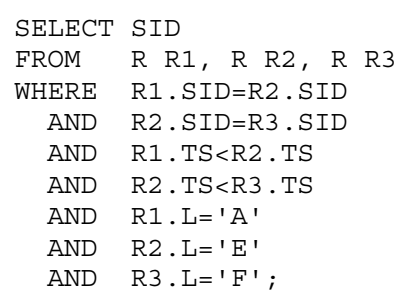

Fig. 1. The relation of data sequences and the content-based sequence retrieval query 
Since in data mining applications databases tend to be very large, there is a problem of appropriate optimizing the database access while performing contentbased sequence retrieval, e.g. by means of the above $S Q L$ query. Database research has developed many indexing techniques, like $\mathrm{B}+$ trees [Com79], bitmapped indexes [O'Neil87], k-d trees [Bent75], R trees [Gutt84], which are used to optimize queries based on exact matches of single tuples. However, these techniques do not significantly improve content-based sequence retrieval queries, which deal with partial matches of multi-tuple sequences. There are also proposals for set-based indexing [MZ98][DP99], which is used to improve subset searching (e.g. find all papers containing "data mining" and "data warehousing" in a keyword list). However, these methods work for retrieval of unordered sets of items only.

In order to realize the shortcomings of the existing indexing methods, let us consider applying $\mathrm{B}+$ tree and set-based indexes to execute the query from Figure 1:

1. Using a $\mathrm{B}+$ tree index, tuples containing all items of each data sequence are joined first (by SID attribute), and then the verification is done whether they contain given items in the given order. This approach can be fairly ineffective since a data sequence may span across many disk block, what results in multiple scanning of each block of the relation.

2. Using a set-based index, the sequence identifiers (SID attribute) of all sequences, which contain the searched items in any order, are found, and then the sequences are read from the relation (perhaps with help of a B+ tree) to verify the ordering of their items. This approach gives much better results, as compared to a $\mathrm{B}+$ tree index, however, the significant overhead comes from reading and verifying the sequences having incorrect ordering.

In this paper we consider content-based retrieval of data sequences from dense databases. By dense databases we mean databases characterized by relatively small number of items, which occur frequently in various order (e.g. web logs), and therefore a set-based index is not efficient. We introduce a new bitmap-oriented indexing method, which optimizes the problem of content-based sequence retrieval. The basic idea behind our method, as compared to set-based indexes, is that the index structure includes not only the items of a sequence, but also the ordering of the items. In this way, we reduce the number of data sequences needlessly read from the database, what results in shorter query execution time. We performed several experiments, which showed the significant improvement over existing indexing methods.

The structure of the paper is as follows. Section 2 describes the sequential index structure and algorithms to create and to use the index. In Section 3 we present the results of our performance experiments. Section 4 contains final conclusions.

\subsection{Basic Definitions and Problem Formulation}

The problem of content-based sequence retrieval can be formulated as follows.

Definition 1.1. Let $L=\left\{l_{l}, l_{2}, \ldots, l_{m}\right\}$ be a set of literals called items. Data sequence $S=\left\langle X_{1} X_{2} \ldots X_{n}\right\rangle$ is an ordered list of sets of items such that each set of items $X_{i} \subseteq L$. $X_{i}$ is called a sequence element. All items in a sequence element are unordered. 
Definition 1.2. We say that a data sequence $\left\langle X_{1} X_{2} \ldots X_{n}\right\rangle$ is contained in another data sequence $\left\langle Y_{1} Y_{2} \ldots Y_{n}>\right.$ if there exist integers $i_{1}<i_{2}<\ldots<i_{n}$ such that $X_{1} \subseteq Y_{i l}, X_{2}$ $\subseteq Y_{i 2}, \ldots, X_{n} \subseteq Y_{i n}$.

Problem formulation. Let $D$ be a database of variable length data sequences. Let $S$ be a data sequence. The problem of content-based sequence retrieval consists in finding in $D$ all data sequences, which contain the data sequence $S$.

\subsection{Related Work}

The problem of mining frequent patterns in databases of data sequences was introduced in [AS95]. In [SA96] the problem was generalized by adding conceptual hierarchies on items and time constraints such as min-gap, max-gap and sliding window. Another formulation of the problem of mining frequently occurring patterns in data sequences was given in [MTV95], where discovered patterns may have different types of ordering: full (serial episodes), none (parallel episodes) or partial and had to appear within a user-defined time window. The episodes were mined over a single data sequence.

Database indexes provided today by most database systems are $\mathrm{B}^{+}$tree indexes to retrieve tuples of a relation with specified values involving one or more attributes [Com79]. Each non-leaf node contains entries of the form $(v, p)$ where $v$ is the separator value which is derived from the keys of the tuples and is used to tell which sub-tree holds the searched key, and $p$ is the pointer to its child node. Each leaf node contains entries of the form $(\mathrm{k}, \mathrm{p})$, where $p$ is the pointer to the tuple corresponding to the key $k$.

A set-based bitmap indexing, which is used to enable faster subset search in relational databases was presented in [MZ98] (a special case of superimposed coding). The key idea of the set-based bitmap index is to build binary keys, called group bitmap keys, associated with each item set. The group bitmap key represents contents of the item set by setting bits to ' 1 ' on positions determined from item values (by means of modulo function). An example set-based bitmap index for three item sets: $\{0,7,12,13\},\{2,4\}$, and $\{10,15,17\}$ is given in Figure 2. When a subset search query seeking for item sets containing e.g. items 15 and 17 is issued, the group bitmap key for the searched subset is computed (see Figure 3). Then, by means of a bit-wise AND, the index is scanned for keys containing 1's on the same positions. As the result of the first step of the subset search procedure, the item sets identified by set $=1$ and $s e t=3$ are returned. Then, in the verification step (ambiguity of modulo function), these item sets are tested for the containment of the items 15 and 17. Finally, the item set identified by set $=3$ is the result of the subset search. Notice that this indexing method does not consider items ordering. 


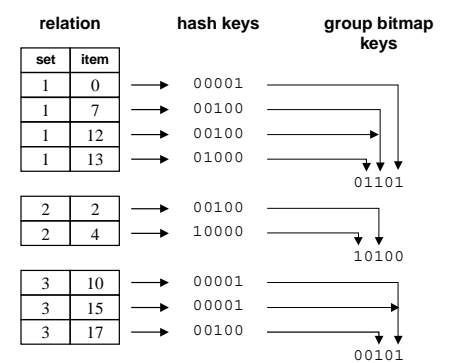

Fig.2. Set-based bitmap index

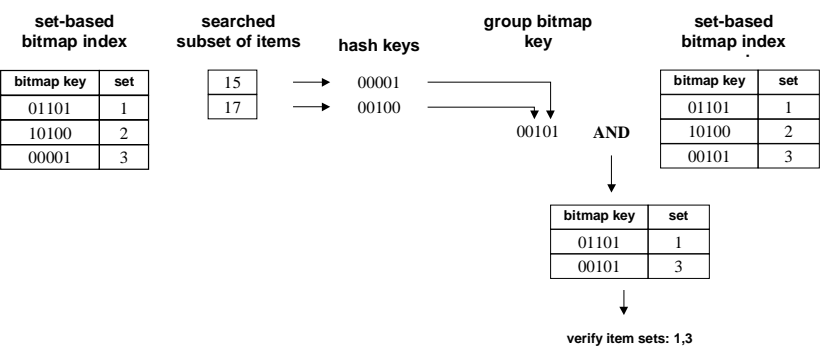

Fig. 3. Set retrieval using set-based bitmap index

In [DP99], a conceptual clustering method, using entropic criterion for conceptual clustering $E C^{3}$ is used to define indexing schemes on sets of binary features. Similar data item sets are stored in the same cluster, and similarity measure based on entropy is used during retrieval to find a cluster containing the searched subset. The method does not consider items ordering.

\section{Sequential Index Structure}

In this Section we present our new indexing method, called sequential indexing, for optimizing content-based sequence retrieval. The sequential index structure consists of sequences of bitmaps generated for data sequences. Each bitmap encodes all items (similarly to a set-based bitmap index) of a portion of a data sequence as well as ordering relations between each two of the items.

The subsection 2.1 contains preliminaries, in the subsection 2.2 we present the index construction algorithm, the subsection 2.3 explains how to use the sequential index structure, finally, in the subsections 2.4 and 2.5 we discuss index storage and maintenance problems.

\subsection{Preliminaries}

Data sequences may contain categorical items of various data types (e.g. character strings, numbers, dates). For sake of convenience, we convert the items to integer values by means of an item mapping function.

Definition 2.1. An item mapping function fi(x), where $x$ is a literal, is a function which transforms a literal into an integer value.

Example. Given a set of literals $L=\{A, B, C, D, E, F\}$, an item mapping function can take the following values: $f i(A)=1, f i(B)=2, f i(C)=3, f i(D)=4, f i(E)=5, f i(F)=6$.

Similarly, we use an order mapping function to express data sequence ordering relations by means of integer values. Thus, we will be able to represent data sequence items as well as data sequence ordering uniformly. 
Definition 2.2. An order mapping function fo( $x, y)$, where $x$ and $y$ are literals and $f o(x, y) \neq f o(y, x)$, is a function which transforms a data sequence $\langle\{x\}\{y\}\rangle$ into an integer value.

Example. For the set of literals used by Definition 2.1 example, an order mapping function can be expressed as: $f o(x, y)=6^{*} f i(x)+f i(y)$, e.g. $f o(C, F)=24$.

Using the above definitions, we will be able to transform data sequences into item sets, which are easier to manage, search and index. An item set representing a data sequence is called an equivalent set.

Definition 2.3. An equivalent set $E$ for a data sequence $S=\left\langle X_{1} X_{2} \quad \ldots \quad X_{n}\right\rangle$ is defined as:

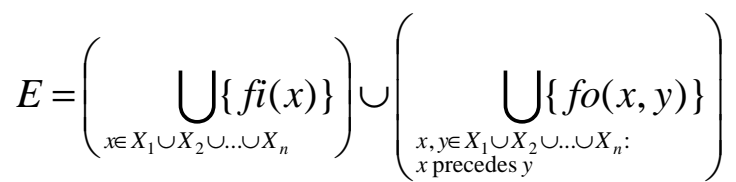

where: $f()_{()}$is an item mapping function and $f o()$ is an order mapping function.

Example. For the data sequence $S=\langle\{A, B\}\{C\}\{D\}\rangle$ and the presented item mapping function and order mapping function, the equivalent set $E$ is evaluated as follows:

$$
\begin{aligned}
E= & \left(\bigcup_{x \in\{A, B, C, D\}}\{f i(x)\}\right)\left(\bigcup_{x, y \text { in }\{<\{A\}\{C\}>,<\{B\}\{C\}>,<\{A\}\{D\}>,<\{B\}\{D\}>,<\{C\}\{D\}>\}}\{f o(x, y)\}\right. \\
= & \{f i(A)\} \cup\{f i(B)\} \cup\{f i(C)\} \cup\{f i(D)\} \cup\{f o(A, C)\} \cup\left\{f_{o}(B, C)\right\} \cup \\
& \cup\{f o(A, D)\} \cup\{f o(B, D)\} \cup\{f o(C, D)\}=\{1,2,3,4,9,15,10,16,22\}
\end{aligned}
$$

Observation. For any two data sequences $S_{I}$ and $S_{2}$, we have: $S_{2}$ contains $S_{1}$ if and only if $E_{1} \subseteq E_{2}$, where $E_{1}$ is the equivalent set for $S_{1}$, and $E_{2}$ is the equivalent set for $S_{2}$. In general, this property is not reversible.

The size of the equivalent set depends on the number of items in the data sequence and on the number of ordering relations between the items. For a given number of items in the data sequence, the equivalent set will be the smallest if there are no ordering relations at all (i.e. $S=\langle X\rangle$, then $|E|=|X|$, since $E=X$ ), and will be the largest if $S$ is a sequence of one-item sets (i.e. $S=\left\langle X_{1} X_{2} \ldots X_{n}\right\rangle$, for all $i$ we have $\left|X_{i}\right|$ $=1$, then $|E|=n+\left(\begin{array}{l}n \\ 2\end{array}\right)$ ).

Since the size of an equivalent set quickly increases while increasing the number of the original sequence elements, we split data sequences into partitions, which are small enough to process and encode.

Definition 2.4. We say that a data sequence $S=\left\langle X_{1} X_{2} \ldots X_{n}\right\rangle$ is partitioned into data sequences $S_{1}=\left\langle X_{1} \ldots X_{a 1}\right\rangle, S_{2}=\left\langle X_{a 1+1} \ldots X_{a 2}\right\rangle, \ldots, S_{k}=\left\langle X_{a j+1} . . X_{n}\right\rangle$ with level $\beta$ if 
for each data sequence $S_{i}$ the size of its equivalent set $\left|E_{i}\right|<\beta$ and for all $x, y \in X_{1} \cup X_{2}$ $\cup \ldots \cup X_{n}$, where $x$ precedes $y$, we have: either $\langle\{x\}\{y\}\rangle$ is contained in $S_{i}$ or $\{x\}$ is contained in $S_{i}$, and $\{y\}$ is contained in $S_{j}$, where $i<j$ ( $\beta$ should be greater than maximal item set size).

Example. Partitioning the data sequence $S=\langle\{A, B\}\{C\}\{D\}\{A, F\}\{B\}\{E\}>$ with level 10 results in two data sequences: $S_{1}=\langle\{A, B\}\{C\}\{D\}\rangle$ and $S_{2}=$ $<\{A, F\}\{B\}\{E\}>$, since the sizes of the equivalent sets are respectively: $\left|E_{l}\right|=9\left(E_{l}=\right.$ $\{1,2,3,4,9,15,10,16,22\})$, and $\left|E_{2}\right|=9\left(E_{2}=\{1,6,2,5,8,38,11,41,17\}\right)$.

Observation. For a data sequence $S$ partitioned into $S_{l}, S_{2}, \ldots, S_{k}$, and a data sequence $Q$, we have: $S$ contains $Q$ if and only if there exists a partitioning of $Q$ into $Q_{1}, Q_{2}, \ldots, Q_{m}$, such that $Q_{1}$ is contained in $S_{i l}, Q_{2}$ is contained in $S_{i 2}, \ldots, Q_{m}$ is contained in $S_{i m}$, and $i_{1}<i_{2}<\ldots<i_{m}$.

Our sequential index structure will consist of equivalent sets stored for all data sequences, optionally partitioned to reduce the complexity. To reduce storage requirements, equivalent sets will be stored in database in the form of bitmap signatures.

Definition 2.5. The bitmap signature of a set $X$ is an $N$-bit binary number created, by means of bit-wise OR operation, from the hash keys of all data items contained in $X$. The hash key of the item $x \in X$ is an $N$-bit binary number defined as follows:

$$
\text { hash_key }(\mathrm{X})=2^{(\mathrm{x} \bmod \mathrm{n})}
$$

Example. For the set $X=\{0,7,12,13\}, N=5$, the hash keys of the set items are the following:

$$
\begin{aligned}
& \text { hash_key (0) }=2^{(0 \bmod 5)}=1=00001 \\
& \text { hash_key(7) }=2^{(7 \bmod 5)}=4=00100 \\
& \text { hash_key(12) }=2^{(12 \bmod 5)}=4=00100 \\
& \text { hash_key(13) }=2^{(13 \bmod 5)}=8=01000
\end{aligned}
$$

The bitmap signature of the set $X$ is the bit-wise OR of all items' hash keys: bitmap_signature $(X)=00001$ OR 00100 OR 00100 OR $01000=01101$

Observation. For any two sets $X$ and $Y$, if $X \subseteq Y$ then:

bitmap_signature $(X)$ AND bitmap_signature $(Y)=$ bitmap_signature $(X)$

where AND is a bit-wise AND operator. This property is not reversible in general (when we find that the above formula evaluates to TRUE we still have to verify the result traditionally).

In order to plan the length $N$ of a bitmap signature for a given average set size, consider the following analysis. Assuming uniform items distribution, the probability that representation of the set $X$ sets $k$ bits to ' 1 ' in an $N$-bit bitmap signature is:

$$
P=\frac{\left(\begin{array}{c}
N \\
k
\end{array}\right) f_{k,|X|}}{N^{|X|}} \text {, where } f_{0,|X|}=0, f_{q,|X|}=q^{|X|}-\sum_{i=1}^{q-1}\left(\begin{array}{l}
q \\
i
\end{array}\right) f_{i,|X|}
$$

Example probabilistic expected value of number of bits set to '1' for a 16-bit bitmap signatures and various set sizes is illustrated in Figure 4. We can observe that e.g. for 
a set of 10 items, $N$ should be greater than 8 (else we have all bits set to 1 and the signature is unusable since it is always matched).

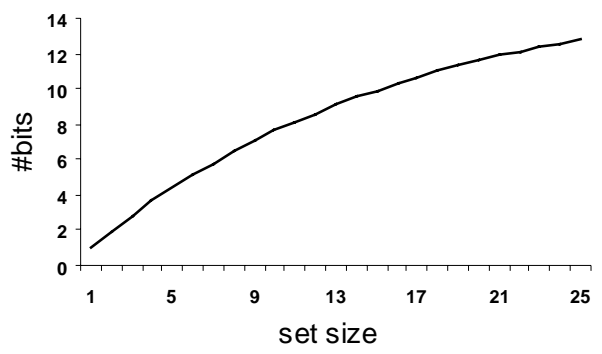

Fig. 4. Number of bitmap signature bits set to ' 1 ' for various set sizes $(N=16)$

The probability that a bitmap signature of the length $N$ having $k 1$ 's matches another bitmap signature of the length $N$ having $m$ 1's is $\left(\frac{m}{k}\right) /\left(\frac{N}{k}\right)$. It means that the smaller $k$, the better pruning is performed during matching bitmap signatures of item sets, in order to check their containment (so we have to verify less item sets).

\subsection{Sequential index construction algorithm}

The sequential index construction algorithm iteratively processes all data sequences in the database. First, the data sequences are partitioned with the given level $\beta$. Then, for each partition of each data sequence, the equivalent set is evaluated. In the next step, for each equivalent set, its $N$-bit bitmap signature is generated and stored in the database. The formal description of the algorithm is given below.

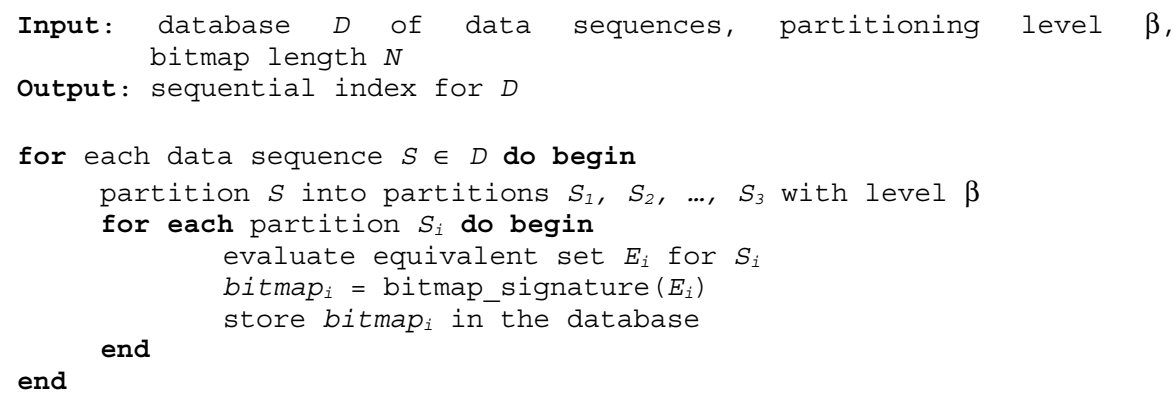

Consider the following example of sequential index construction. Assume that $\beta=10$, $N=16$, and the database $D$ contains three data sequences: $S_{I}=<\{A, B\}\{C\}$ $\{D\}\{A, F\}\{B\}\{E\}\rangle, S_{2}=\langle\{A\}\{C, E\}\{F\}\{B\}\{E\}\{A, D\}\rangle, S_{3}=\langle\{B, C, D\},\{A\}\rangle$.

First, we partition the data sequences with $\beta=10$. Notice that $S_{3}$ is, in fact, not partitioned since its equivalent set is small enough. The symbol $S_{i, j}$ denotes $j$-th partition of the $i$-th data sequence. 


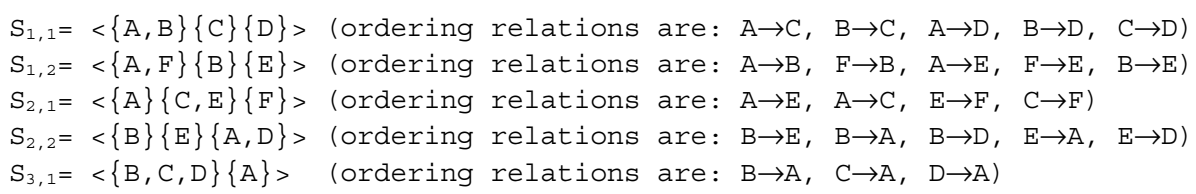

Then we evaluate the equivalent sets for the partitioned data sequences. We use the example item mapping function and order mapping function taken from the definitions 2.1 and 2.2. The symbol $E_{i, j}$ denotes the equivalent set for $S_{i, j}$.

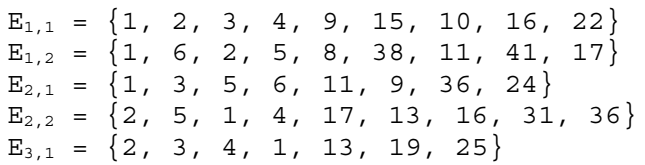

In the next step, we generate 16-bit bitmap signatures for all equivalent sets.

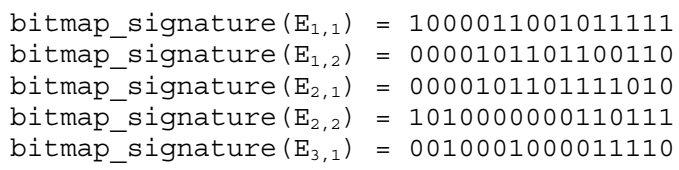

Finally, the sequential index is stored in the database in the following form:

\begin{tabular}{|l|l|}
\hline SID & bitmap_signature \\
\hline 1 & 1000011001011111,0000101101100110 \\
\hline 2 & 0000101101111010,1010000000110111 \\
\hline 3 & 0010001000011110 \\
\hline
\end{tabular}

\subsection{Using Sequential Index for Content-Based Retrieval}

During content-based sequence retrieval, the bitmap signatures for all data sequences are scanned. For each data sequence, the test of a searched subsequence mapping is performed (see observation of Definition 2.4). If the searched subsequence can be successfully mapped to the data sequence partitions, then the data sequence is read from the database. Due to the ambiguity of bitmap signature representation, additional verification of the retrieved data sequence is required. The verification can be performed using the traditional $\mathrm{B}+$ tree method, since it consists in reading the data sequence from the database and checking whether it contains the searched subsequence. The formal description of the algorithm is given below. We use a simplified notation of $Q\left[i_{-}\right.$start..i_end $]$to denote a partition $\left\langle X_{i_{\_} \text {start }} X_{i_{\_} \text {start }+1} \ldots X_{i_{\_} \text {end }}\right\rangle$ of a sequence $Q=\left\langle X_{1} X_{2} \ldots X_{n}\right\rangle$, where $1 \leq i_{-}$start $\leq i \_$end $\leq n$. The symbol \& denotes bit-wise AND operation.

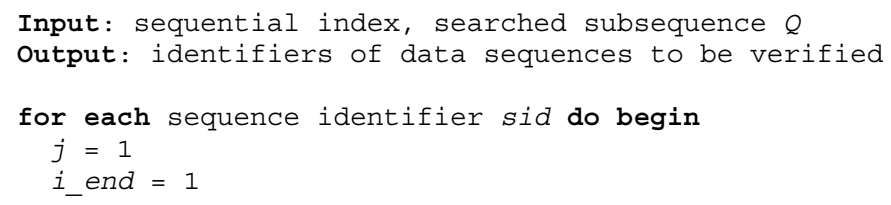




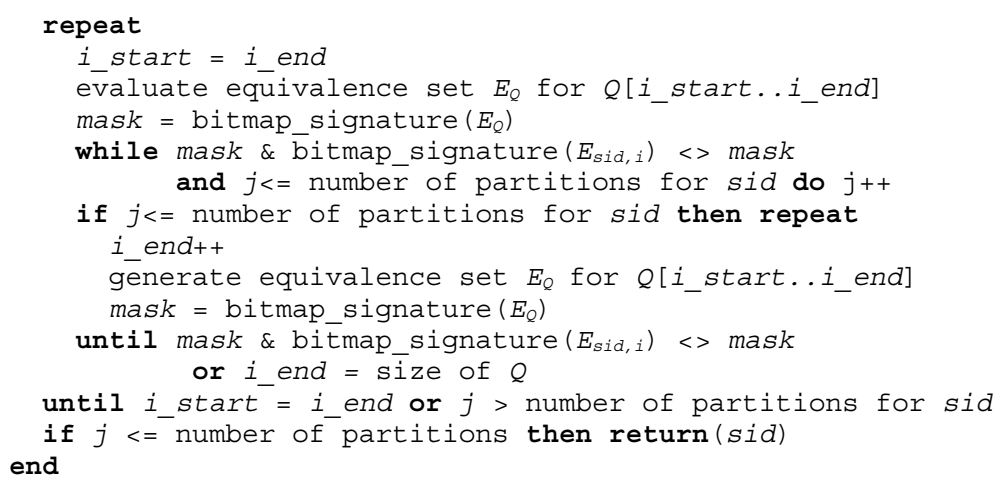

Consider the following example of using sequential index to perform content-based sequence retrieval. Assume that we look for all data sequences, which contain the subsequence $\langle\{F\}\{B\}\{D\}>$. We begin with sid=1. We find that $\langle\{F\}\rangle$ (0000001000000000) matches the first partition (1111101001100001). So, we check whether $\langle\{F\},\{B\}>(0010001000000000)$ also matches this partition. Accidentally it does, but when we try $\langle\{F\},\{B\},\{D\}>(1010101010000000)$, we find that it does not match the first partition. Then we move to the second partition to check whether $\langle\{D\}\rangle(00001000000000000)$ matches the partition (0110011011010000). This test fails and since we have no more partitions, we reject $\operatorname{sid}=1$ (this data sequence does not contain the given subsequence).

In the next step, we check sid=2. We find that $\langle\{F\}\rangle(0000001000000000)$ matches the first partition (0101111011010000). So, we check whether $<\{F\},\{B\}>(0010001000000000)$ also matches this partition. It does not, so we move to the second partition and find that $\langle\{B\}\rangle(0010000000000000)$ matches the partition (1110110000000101). Then we must check whether $\langle\{B\},\{D\}\rangle$ (1010100000000000) also matches the partition. This time the check is positive and since we have matched the whole subsequence, we return sid=2 as a part of the result. The data sequence will be verified later.

Finally, we check sid=3. We find that $\langle\{F\}\rangle(0000001000000000)$ does not match the first partition (0111100001000100). Since we have no more partitions, we reject $s i d=3$ (this data sequence does not contain the given subsequence).

So far, the result of our index scanning is the data sequence identified by sid=2. We still need to read and verify, whether the sequence really contains the searched subset. In our example it does, so the result is returned to a user.

\subsection{Physical Storage}

Since a sequential index is fully scanned each time content-based retrieval is performed, it is critical to store it efficiently. We store index entries in the form of $<p$, $n$, bitmap $_{1}$, bitmap $_{2}, \ldots$, bitmap $_{n}>$, where $p$ is a pointer to a data sequence described by the index entry, $n$ is the number of bitmap signatures, and bitmap ${ }_{i}$ is a single bitmap signature for the data sequence. The pointer $p$ should address the translation table, 
which contains pointers to physical tuples of the relation holding the data sequences (the structure is $\left\langle n, p_{l}, p_{2}, . ., p_{n}\right\rangle$ ). Since we usually have a $\mathrm{B}+$ tree index on a sequence identifier attribute (to optimize joins), we can use its leaves can as a translation table instead of consuming database space by redundant structures. Example storage implementation for the sequential index from Subsection 2.2 is given in Figure 5.

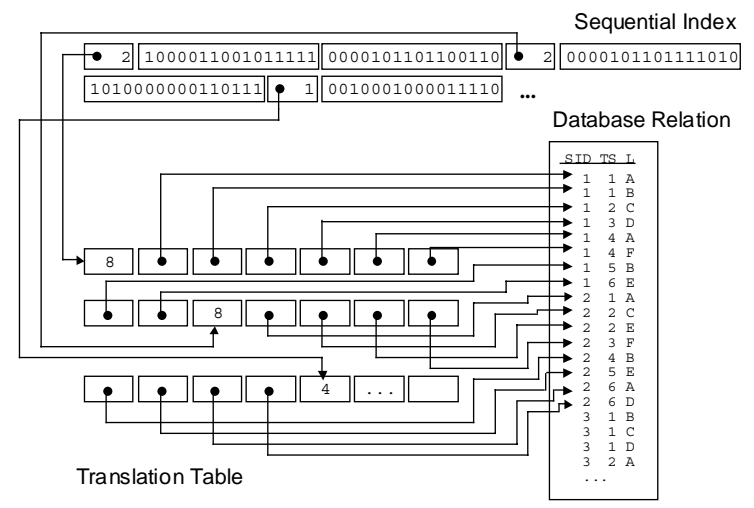

Fig. 5. Example physical storage structure for sequential index

\subsection{Update Operations}

Maintenance of a sequential index is quite expensive, since 1. bitmap signatures are not reversible, and 2. updates may influence partitioning of data sequences. For example, when we insert a new tuple into the database, thus extending a data sequence, we cannot determine what partition should the tuple belong to. Similarly, when we delete a tuple, then both we cannot determine the corresponding partition, and, even if we could do it, we do not know, whether the item being deleted was the only item mapped to a given bit of the bitmap signature (so we could reset the bit).

In order to have a consistent state of a sequential index, we must perform the complete index creation procedure (partitioning, evaluating equivalent sets, generating bitmap signatures) for the data sequence being modified. However, since this solution might reduce DBMS performance for transaction-intensive databases, we propose the following algorithm of offline maintenance for sequential indexes:

1. Whenever a new item is added to an existing data sequence, we set to '1' all bits in the first bitmap signature for the data sequence. It means that any subsequence will match the first bitmap signature, and therefore we will not miss the right one. Any false hits will be eliminated during actual verification of subsequence containment.

2. Whenever an item is removed from an existing data sequence, we do not perform any modifications on the bitmap signatures of the data sequence. We may get false hits, but they will be eliminated during final verification.

Notice that using the above algorithm, the overall index performance may decrease temporarily, but we will not get incorrect query results. Over a period of time, the 
index should be rebuild either completely, or for updated data sequences only, e.g. according to a transaction $\log$.

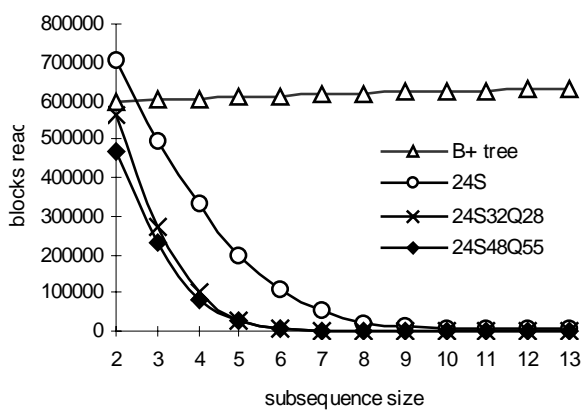

B

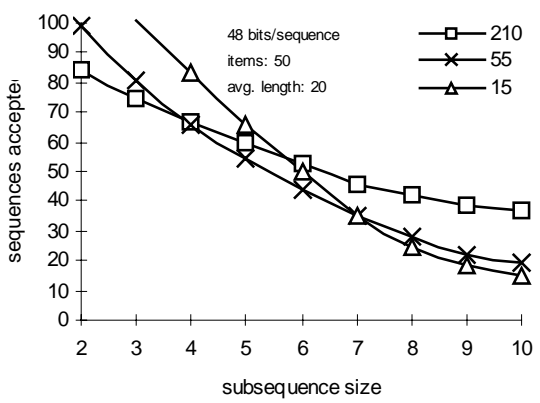

Fig. 6. Experimental results

\section{Experimental Results}

We have performed several experiments on synthetic data sets to evaluate our sequential indexing method. The database of data sequences was generated randomly, with uniform item distribution, and stored by Oracle8 DBMS. We used dense data sets, i.e. the number of available items was relatively small, and therefore each item occurred in a large number of data sequences. The data sequences contained 1-item sets only (pessimistic approach - maximal number of ordering relations).

Figure 6A shows the number of disk blocks (including index scanning and relation access), which were read in order to retrieve data sequences containing subsequences of various lengths. The data set contained 50000 data sequences, having 20 items of 50 in average. The compared database accessing methods were: traditional $S Q L$ query using B+ tree index on SID attribute (B+ tree), 24-bit set-based bitmap index (24S), 32-bit sequential index with $\beta=28$ built on top of 24-bit set-based bitmap index (24S32Q28), and 48-bit sequential index with $\beta=55$ built on top of 24-bit set-based bitmap index (24S48Q55). Our sequential index achieved a significant improvement for the searched subsequences of length greater than 4, e.g. for the subsequence length of 5 we were over 20 times faster than the $\mathrm{B}+$ tree method and 8 times faster than the set-based bitmap index.

We also analyzed the influence of the partitioning level $\beta$ value on the sequential index performance. Figure 6B illustrates the filtering factor (percentage of data sequences matched) for three sequential indexes built on bitmap signatures of total size of 48 bits, but with different partitioning. We noticed that partitioning data sequences into a large number of partitions ( for long subsequences, but worsens the performance for short subsequences. Using a small number of data sequence partitions (high $\beta$ ) results in more "stable" performance, but the performance is worse for long subsequences. 


\section{Final Conclusions}

Content-based sequence retrieval is specific in the sense that it requires complicated $S Q L$ queries and database access methods (multiple joins, inefficient optimization). In this paper we have introduced the new indexing method, called sequential indexing, which can replace a $\mathrm{B}+$ tree indexing and set-based indexing when searched databases of data sequences are dense. During experiments, we have found that the most efficient solution is to combine a set-based index (which checks items of a data sequence) with a sequential index (which checks the items ordering), what results in dramatic outperforming $\mathrm{B}+$ tree access methods. Application areas of the sequential indexing method include e.g. searching web access paths, searching sequential patterns stored in databases, discovery of sequential patterns, sequential data validation.

\section{Bibliography}

[AS95] Agrawal, R., Srikant, R., Mining Sequential Patterns, Proc. $11^{\text {th }}$ Int'l Conf. Data Engineering, 1995

[Bay98] Bayardo R. J., Efficiently Mining Long Patterns from Databases, Proc. of the ACM SIGMOD International Conference on Management of Data, 1998

[Bent75] Bentley, J.L., Multidimensional binary search trees used for associative searching, Comm. of the ACM 18

[Com79] Comer D., The Ubiquitous B-tree, Comput. Surv. 11, 1979

[DP99] Diamantini, C., Panti, M., A Conceptual Indexing Method for Content-Based Retrieval, Proc. of the $15^{\text {th }}$ IEEE Int'l Conf. on Data Engineering, 1999

[Gutt84] Guttman, A., R-trees: A dynamic index structure for spatial searching, Proc. of ACM SIGMOD International Conf. on Management of Data, 1984

[GWS98]Guralnik V., Wijesekera D., Srivastava J., Pattern Directed Mining of Sequence Data, Proc. of the 4th Int'l Conference on Knowledge Discovery and Data Mining, 1998

[MT96] Mannila H., Toivonen H., Discovering generalized episodes using minimal occurrences, Proc. of the 2nd Int'l Conf. on Knowledge Discovery and Data Mining , 1996

[MTV95]Mannila H., Toivonen H., Verkamo A.I., Discovering frequent episodes in sequences, Proc. of the 1st Int'l Conference on Knowledge Discovery and Data Mining , 1995

[MZ98] Morzy, T., Zakrzewicz, M., Group Bitmap Index: A Structure for Association Rules Retrieval, Proc. of 4th International Conference on Knowledge Discovery and Data Mining, AAAI Press, New York, 1998

[O'Neil87] O'Neil, P, Model 204 Architecture and Performance, Springer-Verlag Lecture Notes in Computer Science 359, 2nd International Workshop on High Performance Transactions Systems (HTPS) 1987, Asilomar, CA

[SA96] Srikant R., Agrawal R., Mining Sequential Patterns: Generalizations and Performance Improvements, Proc. of the 5th Int'l Conf. on Extending Database Technology, 1996 